\title{
Measures of network vulnerability indicators for risk evaluation and exposure reduction
}

\author{
M. Di Gangi \& A. Luongo \\ Dip.to di Architettura Pianificazione ed Infrastrutture di Trasporto, \\ Università degli Studi della Basilicata, Potenza, Italy
}

\begin{abstract}
Reliability of transportation systems can be affected by events both exogenous to the system (i.e. earthquakes, floods, terrorism attack, etc.) and endogenous to the system (i.e. dangerous freight, etc.). Hazardous events can introduce disconnections in transportation systems whose effects can be relevant both in the short term (difficulties in evacuation procedures and aids to population) and in the long term (effects in local services and local economies). For these main reasons, in particular considering nonurban situations, to guarantee an acceptable level of accessibility among urban centers an analysis of network vulnerability is relevant. In this paper, after an introduction concerning the concept of network vulnerability, it is proposed an enhanced method, based on consolidated procedures generally adopted in the analysis of transport networks, to explore a transport network in order to put in evidence weakness in some of the links by computing quantitative indicators. The proposed methodology can be adopted, once defined a risk scenario, both to select where to plan necessary adjustments on transport system and to define adequate operations for evacuation and to come to the aid of population, in order to reduce exposure. An explicative application to a real system is also shown to put in evidence capabilities of the proposed indicators.
\end{abstract}

Keywords: network vulnerability, risk analysis.

\section{Introduction}

A large amount of studies concerning vulnerability of single structures can be found in literature, whilst more rarely such studies concern vulnerability of either transportation or territorial systems. 
Aims of a vulnerability reduction policy are to favour emergency management in order to guarantee timely aids to victims and an orderly and gradual evacuation of population, taking care in avoiding congestion phenomena on links.

As a matter of fact interventions to reduce vulnerability of a transportation system allow not only to enhance the capacity to evacuate a certain area if a hazardous event occurs but also to reduce those accessibility problems that can occur in certain settlements

Reliability of a system can be defined as the capability to work properly, within a certain period of time and in the predicted conditions. This, for transportation system, can be represented, in normal conditions, as the possibility to connect each other the several origins and destinations of displacements and, in emergency conditions, as the guarantee that links are able to bear stresses and flows due to evacuation avoiding congested situations.

In this paper, after a short description of methodological framework, it is presented an analytical procedure to evaluate quantitative measures that can be used both to compute the degree of reliability of connections of the several origin-destination pairs and to quantify the potential exposure due to the vulnerability of the network. Furthermore the results of some applications to real cases are also reported.

\section{Methodological framework}

An increase in the interest in road network reliability by scientific community can be observed since 1990s. In particular, after the seismic event of Kobe in 1995, a significant interest has been concentrated in those aspects concerning reliability of transportation network.

Several definitions and classifications of reliability have been proposed in literature [1]. A first classification of the studies conducted on transportation network reliability can be done between those methodologies considering only supply system and those ones that take into account also interaction between demand and supply.

Within the first case it is possible to distinguish two aspects: in the first one, concerning connectivity reliability $[2,3]$, analytical procedures are proposed to individuate reliability of connections; moreover D'Este and Taylor [4] suggest some indicators to evaluate weakness points of a network. The second one take into account capacity reliability $[5,6]$ defined as the probability that a network, whose state may be normal or degraded, is able to satisfy a certain level of demand that is can accept a fixed traffic volume. This probability is defined by Chen et al. [5] as the probability that residual capacity of a network results, once fixed the capacity loss due to degradation, greater than or equal to the required demand.

In the latter case reliability of travel times has been considered, defined as the probability that a journey between an origin-destination pair is concluded within a definite time interval [2]. To evaluate travel time reliability several measure methodologies have been proposed $[7,8]$. 
Starting from the concept of network weakness proposed by D'Este and Taylor [4], this work develops the concept of robustness of connections proposed by the author [9], taking advantage of the properties of an assignment model recently proposed by Russo and Vitetta [10], and allows the definitions quantitative measures usable both to evaluate a degree of reliability of connections among the several origin-destination pairs and to quantify the level of potential exposure due to the network vulnerability.

A particular evidence should be put on the concept of network vulnerability connected to the consequences of the lapse of performance of one (or more) of the links that make it up, in terms of impact on the whole territorial system served.

\section{The proposed approach}

Starting from the application of the concept of vulnerability to the existing connection of a generic origin-destination (in the following o/d) pair, it is possible to develop the analysis to the access from a particular site to the network and, finally, to analyses that can involve the whole network.

A first definition of point (or node) vulnerability can be given as [4]: "a node is vulnerable if loss (or substantial degradation) of a small number of links significantly diminishes the accessibility of the node, as measured by a standard index of accessibility".

By means of this definition, vulnerability can be defined in terms of the quality of accessibility from a given node to the remaining part of the network.

One of the aims of the proposed vulnerability analysis is to estimate those points constituting a weakness for the network where possible interruptions and/or damages can cause unpredictable effects on the performances of the whole network and, consequently, give some indications on actions to carry out to strengthen the level of connection.

An outline of dependencies among the several operative tasks in which the proposed approach can be divided is shown in Figure 1. As it can be seen, a first analysis can be conducted exclusively on the supply model of the considered transportation system leaving aside the demand.

Subsequently, starting from results on the reliability analysis of the supply system and introducing evaluations on the involved demand, passing through the definition of a risk scenario, it is possible to analyse the exposure of the considered network.

In the following activities to be developed in order to conduct the described analyses are described; they can be summarised as follows:

- definition of the type of the analysis;

- individuation of suitable variables (indicators) able to quantify the level;

- measure of values assumed by considered indicators. 


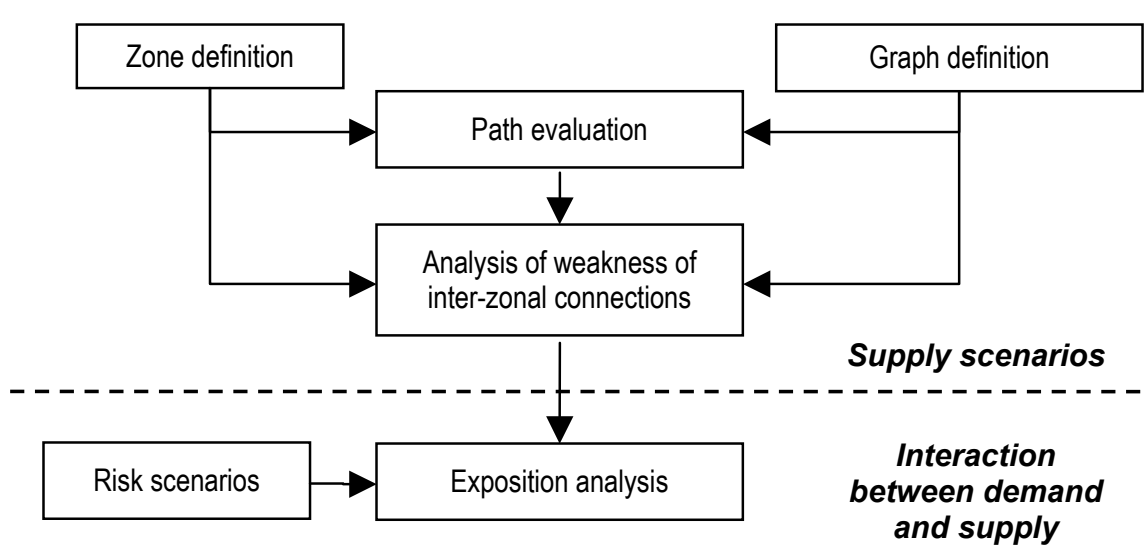

Figure 1: Dependencies among the several operative tasks of the proposed procedure.

\subsection{Deterministic measures}

A first approach for the analysis of weakness of connections can be based on the evaluation of connectivity in terms of existence and characteristics of alternative paths connecting the considered zones.

Let $N$ be the set of nodes, $L$ the set of links and $G(N, L)$ the graph representing the considered transportation system. Let the generic o/d pair od connected by a number $N_{o d}^{k}$ of paths that make up the set $K_{o d}$; let $a_{k}$ be the number of links that compose the generic path $k \in K_{o d}$. Let define an incidence link-path matrix $\Delta$, whose generic element $\delta_{i j}{ }^{k}$ is equal to 1 if link $i j$ belongs to path $k$ and 0 otherwise, and an incidence link-o/d pair matrix $\Gamma$ whose generic element $\gamma_{i j}^{o d}$ is equal to 1 if link $i j$ belongs at least to one of the paths connecting the pair $o d$, otherwise it is equal to 0 .

Following these definitions, once fixed an o/d pair od, $n_{o d}^{K}=\Sigma_{k \in K r} a_{k}$ represents the total number of links belonging to the paths connecting the considered pair and $n_{o d}^{G}=\Sigma_{i \in N} \gamma_{i j}^{o d}$ represents the number of links of the graph crossed by the paths connecting the considered pair.

It can be introduced, as synthetic measure, a Weakness Index $\left(W I_{o d}\right)$ of the connections defined as follows:

$$
\begin{array}{ll}
W I_{o d}=\frac{\frac{n_{o d}^{K}}{n_{o d}^{G}}-1}{N_{o d}^{k}-1} & \text { if } N_{o d}^{k}>1 \\
W I_{o d}=1 & \text { if } N_{o d}^{k}=1
\end{array}
$$


This indicator, whose values are between 0 and 1 , is related to each o/d pair and give information on the overlapping among the paths connecting the considered pair. A value $W I_{o d}=0$ means that to each link belonging to a path corresponds a unique infrastructural section that is different paths are absolutely not overlapped each other. Otherwise, a value $W I_{o d}=1$ implies that all the paths are exactly overlapped and coincide to one path.

So, the closer to zero is the value of this indicator the more different are the paths and the more the connectivity of the considered o/d pair improves; that is weakness due to events that could cause impassability of some road section reduces. To compute such indicator it is necessary to evaluate a set of paths connecting the considered o/d pairs; path evaluation can be conducted by means of a k-path algorithm taking into account of network characteristics.

\subsection{Stochastic measures}

In the approach that follows, the computation of paths is performed introducing probabilistic path choice models and, in particular, C-Logit formulation proposed by Cascetta et al. [11]. In such approach path choice is based on the mathematical framework of a multinomial Logit model with a modification in systematic utility given by:

$$
V_{k}^{*}=V_{k}-C F_{k}
$$

where $C F_{k}$, defined as commonality factor of path $k$, takes into account of the overlapping of path $k$ with all the other paths belonging to the set $K_{o d}$ connecting the o/d pair od.

In particular, considering the D-C-Logit model developed by Russo and Vitetta [10], for each link $i j$ crossed by effective paths connecting the o/d pair $o d$ it is possible to evaluate link choice probability as:

$$
p_{i j, o d}=w_{i j, o d} / \Sigma_{m \in B(j)} w_{m j, o d}
$$

where $w_{i j, o d}$ represents the link weight, depending on the considered pair od and $B(j)$ represents the set of the initial nodes of those links entering node $j$ (the backward star of node $j$ ).

In order to determine such weight, the link multiplicity $N_{i j, o d}$ of link $i j$ respect pair $o d$ is defined, it indicates the number of paths crossing link $i j$ that connect $\mathrm{o} / \mathrm{d}$ pair $o d$.

Starting from the definition of multiplicity for each link, it is possible to evaluate the total number of paths $N^{k}$ co connecting pair od as:

$$
N_{o d}^{k}=\Sigma_{m \in B(d)} N_{m d, o d}
$$

where $B(d)$ represents the set of the initial nodes of those links entering destination node $d$.

The total number of links building up the set of paths connecting the o/d pair $\left(n^{K}{ }_{o d}\right)$ is computed by cumulating, for all the links belonging to the paths, the values of multiplicity $N_{i j, o d}$ of link $i j$ respect o/d pair od: 


$$
n_{\text {od }}^{K}=\Sigma_{i j} N_{i j, o d} \quad \forall i j: p_{i j, o d} \neq 0
$$

Furthermore the number $n_{o d}^{G}$ of links of the graph involved by paths connecting the same o/d pair is given by the cardinality of the set of links belonging to paths:

$$
n_{o d}^{G}=\left\|\left\{i j: p_{i j, o d} \neq 0\right\}\right\|
$$

Weakness Index $\left(W I_{o d}\right)$ can be then computed and it is possible to define, for each link and each o/d pair, another indicator taking into account not only the number of paths connecting the considered o/d pair crossing the link but also the link choice probability referred to each path connecting the considered o/d pair. This yields to a Weighted Weakness Index $\left(W W I_{i j, o d}\right)$ where the ratio between multiplicity $N_{i j, o d}$ of link $i j$ concerning o/d pair od and the total number of paths $N^{k}{ }_{o d}$ connecting pair $o d$ is weighted with the link choice probability $p(i j / j)$ as follows:

$$
W W I_{i j, o d}=\frac{N_{i j, o d} p_{i j, o d}}{N_{o d}^{k}}
$$

If for each o/d pair $\max _{i j}\left\{W W I_{i j, o d}\right\}$ is evaluated, it is possible to consider as more at risk (referring to the considered o/d pair) the link for which such value occurs. In order to obtain a synthetic link indicator referred to the whole set of o/d pairs, a Link Weakness Index $\left(L W I_{i j}\right)$ is built up similarly to the previous one by considering for each link the values of all the o/d pairs:

$$
L W I_{i j}=\frac{\Sigma_{o d} N_{i j, o d} p_{i j, o d}}{\Sigma_{o d} N_{o d}^{k}}
$$

This indicator allows one to put in evidence all those infrastructural sections more interested by the considered o/d pairs and can be used to define a hierarchy among the sections to find out those ones whose working conditions and effectiveness are essential to preserve network connectivity.

Using the parameters above defined and considering not only the vulnerability among connections but also the potential exposure of the network, it is necessary to take into account the level of demand existing among the several o/d pairs. So, a first approach indicator of potential exposure for each link can be introduced by weighting previously defined Link Weakness Index $\left(L W I_{i j, o d}\right)$ values with the demand flow $d_{o d}$ interesting each o/d pair, obtaining a Link Exposure Index $\left(L E I_{i j}\right)$ :

$$
L E I_{i j}=\frac{\Sigma_{o d} W W I_{i j, o d} \cdot d_{o d}}{\Sigma_{o d} d_{o d}}
$$

Also this indicator can be used in ranking links, on the basis of the level of the potential exposure associated to each one of them, to define priority rules for those operations aimed to the protection and safeguard of the network. 


\section{Applicative example}

The aim of the example here shown is to individuate the connectivity level existing within the territory of the province of Potenza (Fig. 2), located in South Italy that, for its particular nature, is subjected to natural hazards such as landslides and earthquakes.
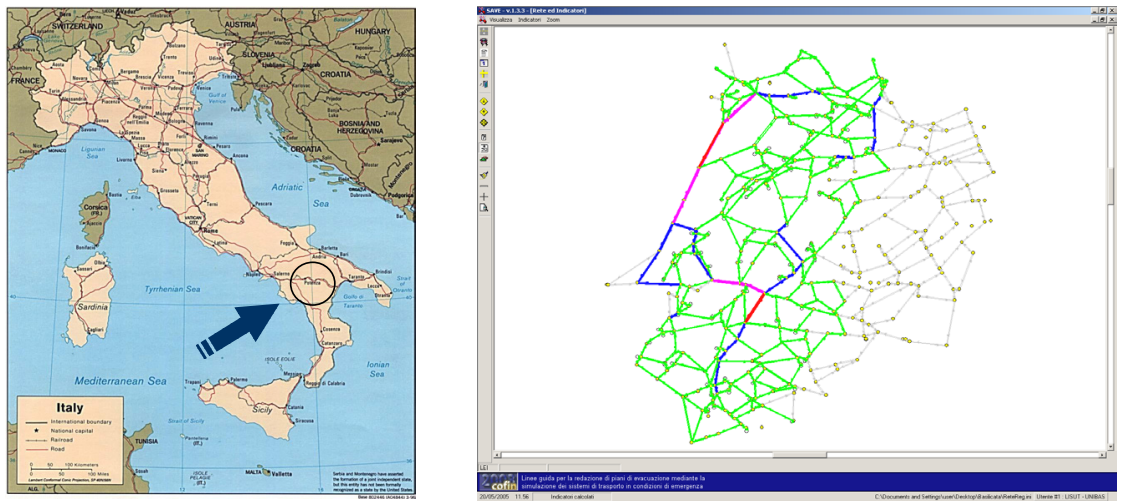

Figure 2: Location of the province of Potenza and graph of the provincial road network.

The considered area has been divided into 100 zones, coinciding with the territory of municipalities, and the definition of the road network has been conducted by considering the main road sections up to the provincial level. Link travel time has been evaluated by assuming an average speed for each link depending on both physical and functional characteristics of the road section that it represents. Main characteristics of the graph of the road network are summarised in Tab. 1.

Table 1: $\quad$ Main characteristics of the graph of the road network.

\begin{tabular}{cccc}
\hline $\begin{array}{c}\text { Number } \\
\text { Of zones }\end{array}$ & $\begin{array}{c}\text { Number } \\
\text { of nodes }\end{array}$ & $\begin{array}{c}\text { Number } \\
\text { of links }\end{array}$ & $\begin{array}{c}\text { Number of } \\
\text { O/D pairs }\end{array}$ \\
\hline 100 & 397 & 1156 & 9900 \\
\hline
\end{tabular}

In the following are reported the main results obtained by applying the proposed procedure to the above described network. The aim of this example is only to show one of the possible use of the proposed indicators. For this reason all those particular comments on the results obtained, such as identifiyng the section of the network corresponding to the links of the graph, are not reported.

\subsubsection{Computation of indexes}

The proposed set of indicators has been evaluated on the provincial road network. First of all, the number $n^{K}{ }_{\text {od }}$ of paths for each O/D pair has been deducted and classified. 
Table 2: $\quad$ Distribution of the number of paths for each $\mathrm{O} / \mathrm{D}$ pair.

\begin{tabular}{ccccccccc}
\hline $\boldsymbol{n}_{\text {od }}^{K}$ & 1 & $2-5$ & $6-10$ & $11-20$ & $21-50$ & $51-100$ & $>100$ & \\
\hline O/D & 2904 & 5062 & 1202 & 454 & 213 & 51 & 14 & 9900 \\
Pairs & $29.3 \%$ & $51.1 \%$ & $12.1 \%$ & $4.6 \%$ & $2.2 \%$ & $0.5 \%$ & $0.1 \%$ & $100.0 \%$ \\
\hline
\end{tabular}

As it can be seen from values shown in Tab. 2, more that the $90 \%$ of $\mathrm{O} / \mathrm{D}$ pairs result connected by a number of paths less than 10 and about the $29 \%$ of the O/D pairs have only one path connecting them.

Then results obtained in terms of Weakness Index for the considered O/D pairs have been classified and summarised in Tab. 3. As it can be seen, the same $\mathrm{O} / \mathrm{D}$ pairs connected by only one path show a value for this measure equal to 1 , that is present the highest level of weakness.

Table 3: $\quad$ Distribution of the Weakness Index for each O/D pair.

\begin{tabular}{ccccccccccccc}
\hline WI & $=0$ & $0-.1$ & $.1-.2$ & $.2-.3$ & $.3-.4$ & $.4-.5$ & $.5-.6$ & $.6-.7$ & $.7-.8$ & $.8-.9$ & $.9-1$ & $=1$ \\
\hline n. of & 22 & 12 & 101 & 578 & 890 & 995 & 1213 & 1621 & 1124 & 440 & 0 & 2904 \\
pairs & $0.2 \%$ & $0.1 \%$ & $1.0 \%$ & $5.8 \%$ & $9.0 \%$ & $10.1 \%$ & $12.3 \%$ & $16.4 \%$ & $11.4 \%$ & $4.4 \%$ & $0.0 \%$ & $29.3 \%$ \\
\hline
\end{tabular}

From the shown indicators, together with the WWI indicator, considerations on weakness (or robustness) of connectivity among the several O/D pairs can be made.

From the operational point of view, it is also important to define not only what kind of interventions must be done to reduce weaknes but, especially in a context either of limited resources or of priorities, it is really important to individuate where such interventions should be conducted. Hence, one of the main purposes can be to identify those links whose "weight", in terms of guaranteed connectivity, is more relevant.

Information on the role assumed by each link of the network in assuring connectivity among the O/D pairs can be obtained by the above defined Link Weakness Index $\left(L W I_{i j}\right)$. As a matter of fact all those links of the graph, corresponding to infrastructural sections, that are more interested by the considered O/D pairs can be put in evidence. Referring to the test network here introduced, it is possible to obtain a classification of links, as shown in Tab. 4.

In particular, it is also possible to go more in depth and explicitly identify those links whose contribution to the weakness of the whole network is more relevant, rearranging the set of links in a ordered list those critical links whose level of service must be protected in order to guarantee connectivity among O/D pairs, as shown in Fig. 3.

Table 4: $\quad$ Distribution of the Link Weakness Index for each link of the network.

\begin{tabular}{cccccccc}
\hline LWI & $=0$ & $0-.03$ & $.03-.06$ & $.06-.09$ & $.09-.12$ & $.12-.15$ & $.15-.25$ \\
\hline n. of & 284 & 764 & 56 & 24 & 8 & 16 & 4 \\
links & $24.5 \%$ & $66.1 \%$ & $4.8 \%$ & $2.1 \%$ & $0.7 \%$ & $1.4 \%$ & $0.4 \%$ \\
\hline
\end{tabular}




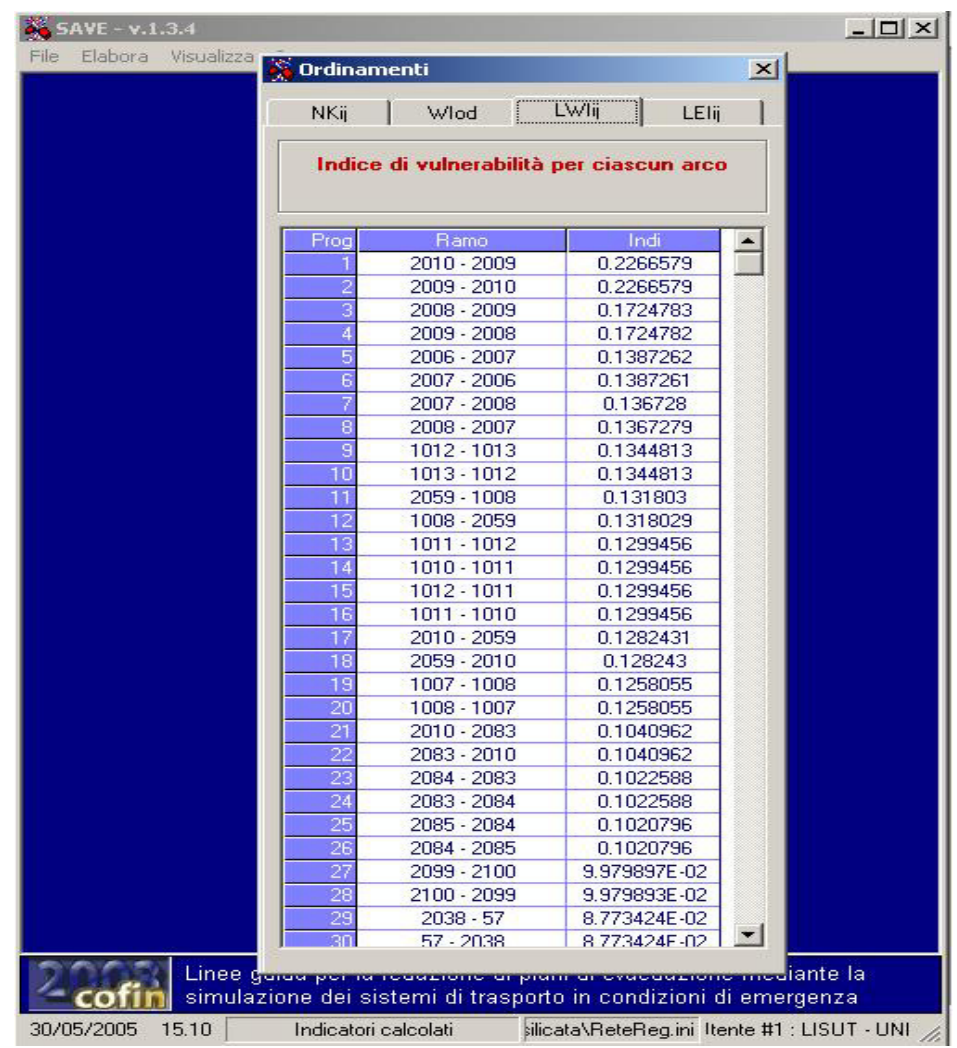

Figure 3: Example of the output of the ranking procedure among the link of the road network.

Similarly, it is possible to obtain an ordered list of links in terms Link Exposure Index $\left(L E I_{i j}\right)$, once the level of demand for each O/D pair is introduced.

\section{Some considerations}

The method proposed in this work, based on consolidated procedures generally adopted in the analysis of transport networks, exploring a transport network can put in evidence network weakness by means of the computation of the defined quantitative indicators.

As briefly shown in the example, it is given a possible way, once defined a risk scenario in terms of supply system and demand, to identify those critical sections of the transport system where necessary adjustments must be planned to guarantee level of service of the network. This is very important expecially in those cases where a good functionality of the network is critical, as those events of evacuation or coming to aid of population, in order to reduce exposure. 


\section{References}

[1] Berdica, K. An introduction to road vulnerability - what has been done, is done and should be done. Transport Policy, vol. 9, no. 2, 117-127, 2002

[2] Bell, M. G. H., Iida, Y. Transportation network analysis. John Wiley \& Sons, Chichester, 1997

[3] Iida, Y. Basic concepts and future direction of road network reliability analysis. J. Adv. Transpn., 33(2), 125-134, 1999

[4] D’Este, G. M., Taylor, M. A. P. Network vulnerability: an approach to reliability analysis at the level of national strategic transport networks. In The Network Reliability of Transport, (M. G. H. Bell \& Y. Iida, eds.) pp. 23-44, Pergamon, 2003

[5] Chen, A., Yang, H., Lo, H. K., Tang, W. H. A capacity related reliability for transportation networks. J. Adv. Transpn., 33(2), 183-200, 1999

[6] Yang, H., Lo, H. K., Tang, W. H. Travel time versus capacity reliability of a road network. In Reliability of Transport Network, (M. G. H. Bell \& C. Cassir, eds.), Research Studies Press, Baldock, 2000

[7] Asakura, Y., Kashiwadani, M. Road network reliability caused by daily fluctuation of traffic flows. Proc. 19th PTRC Summer Annual Meeting, University of Sussex, Brighton, July, 73-84, 1991.

[8] Inouye, H. An evaluation of the reliability of travel time in road networks based on stochastic user equilibrium. In The Network Reliability of Transport, (M. G. H. Bell \& Y. Iida, eds.) pp. 79-91, Pergamon, 2003

[9] Di Gangi, M., Luongo A.S. Definizione della vulnerabilità di un sistema di trasporto in condizioni di emergenza: indicatori quantitativi per l'analisi sperimentale. Presented at VII annual seminar "Metodi e tecnologie dell'ingegneria dei trasporti", Reggio Calabria, 2-3 Dec.2004

[10] Russo, F., Vitetta, A. An assignment model with modified Logit, which obviates enumeration and overlapping problems. Transportation 30177 201, 2003

[11] Cascetta, E., Nuzzolo, A., Russo, F., Vitetta A. A modified logit route choice model overcoming path overlapping problems, specification and some calibration results for interurban networks. In Transportation and Traffic Theory (J.B. Lesort editor), Pergamon, 1997 